\title{
A case report of a delayed-interval delivery in a dichorionic diamniotic twin pregnancy
}

\author{
Justin White $\mathrm{BSc}^{1}$, Catherine Craig MD², Diane Ahn MD² \\ 'Class of 2016, Faculty of Medicine, Dalhousie University \\ ${ }^{2}$ Department of Obstetrics and Gynecology, IWK Health Centre, Dalhousie University
}

\begin{abstract}
Background: Twin gestation carries the risk of preterm delivery, which can result in neonatal morbidity and mortality. A delayed-interval delivery is a rare option when it is believed delayed arrival of the second twin will improve its outcome, provided there are no contraindications to the procedure.
\end{abstract}

Case: Dichorionic diamnotic twins presented at $23 \mathrm{w} 2 \mathrm{~d}$ with previous preterm premature rupture of membranes in twin A, which resulted in spontaneous vaginal delivery of twin A at 26 wld followed by a delayed-interval delivery of twin B, eight days later at 27 w2d via caesarean section.

Conclusion: Since there are no guidelines on the procedure, case series and reports are a main source of information. Although successful outcomes have been documented, it is essential to explain the maternal, fetal and neonatal risks and benefits fully to the patient.

M ultiple pregnancies are often impacted by conditions that can lead to preterm delivery and thus high morbidity and mortality of the neonates involved. In 2006, approximately $60 \%$ of twins and $92.6 \%$ of triplets were born before 37 weeks gestational age. ${ }^{1}$ Perinatal mortality rates in developed countries range from 47 to 120 per 1000 births for twins and 93 to 203 per 1000 births for triplets. ${ }^{2}$ Morbidities associated with prematurity include respiratory failure, pneumonia, congenital malformations, necrotizing enterocolitis, intraventricular hemorrhage and retinopathy of prematurity, as well as long term deficits such as cerebral palsy and neurological and motor dysfunction. ${ }^{3}$ Multifetal pregnancy also exposes the mother to increased risk of preeclampsia, placental abruption, post-partum hemorrhage, gestational hypertension and operative delivery among other obstetrical complications. ${ }^{4}$

The developed world, including Canada, has experienced a dramatic increase in the number of twin pregnancies. There has been a $35 \%$ increase in the birth of twins since $1974 . .^{5}$ Advanced maternal age, low maternal age, as well as the use of assisted reproductive technologies (ART) have contributed to this rise. ${ }^{6}$ One theory as to why older mothers are likely to experience multifetal pregnancies is that advanced maternal age is associated with increased levels of circulating follicle stimulating hormone (FSH) resulting in multiple ovulations due to multiple follicle growth. ${ }^{7}$ In addition, ART often involves the implantation of more than one blastocyst in the uterus with the intention of a singleton pregnancy. However, more than one blastocyst may survive. ${ }^{5}$

Although most preterm twin births result in delivery of both fetuses over a short interval, there are cases where the uterus will stop contracting after the first delivery. ${ }^{8}$ Given the absence of fetal distress, infection, congenital malformation or placental abruption, this provides an opportunity where delivery of the second fetus can be delayed in order to increase survival rate and decrease morbidity of prematurity of the second twin, assuming a dichorionic diamniotic pregnancy. This method is referred to as a delayedinterval delivery. ${ }^{9}$ The amount of evidence supporting delayed-interval deliveries is minimal as no randomized trial has been conducted. Due to the high-risk nature of such deliveries, obstetricians are forced to focus more on achieving positive outcomes on a case per case basis rather than on a collective research level. ${ }^{9}$ This is why a large portion of the evidence is based on synthesized literature from case reports and many of the reviews published use the same case reports. Furthermore, there is a large sampling bias whereby unsuccessful delayed-interval deliveries are not reported. There is currently no consensus regarding technique nor is there statistical significance in techniques currently used. ${ }^{8}$

A delayed-interval delivery is indicated when it is believed the delayed arrival of the second twin will improve its outcome. Neonatal survival after preterm delivery correlates directly with the number of days in utero from gestational ages 23 through 28 weeks. ${ }^{10}$ If the birth of the first twin leads to the delivery of the second twin, it contributes to perinatal morbidity and mortality. Survival to discharge of twins after delivery at 22-23, 24, and 25 weeks are $11 \%, 23 \%$, and $51 \%$, respectively. At such extremes of viability, even small gains in gestation can have significant impacts on survival. ${ }^{11}$ Roman et al. reported neonatal outcomes among 16 retained fetuses who survived their NICU 
stay post delivery of twin A at $16 \mathrm{w} 3 \mathrm{~d}$ to $25 \mathrm{w} 5 \mathrm{~d}$. The associated neonatal morbidity included respiratory distress syndrome (75\%), major infection (37.5\%), bronchopulmonary dysplasia (12.5\%) and $37.5 \%$ of the neonates actually survived without major morbidity. There were no cases of necrotizing enterocolitis or grade III/IV intracranial hemorrhage. ${ }^{12}$ Indeed, the survival rate increases markedly for neonates born from 22-25 weeks gestational age. Data suggests that of neonates born between 23-25 weeks gestational age, $40 \%$ will have a normal neurodevelopmental outcome. At 25 weeks gestational age, there is an $80 \%$ chance of survival with a NICU admission. ${ }^{13}$ Farkouh et al. demonstrated that the retained twin had a shorter hospital admission with a mean length of stay of 47 days compared to 86 days for the first born twin in their study. ${ }^{14}$

However, there are several contraindications to delayed-interval delivery, which must be respected. Gestational age beyond 30 weeks generally has positive outcomes and, therefore, does not necessitate the need to delay delivery. Monochorionicity is contraindicated as vascular anastomosis in the retained placenta may increase the risk of complications, however, it has been performed successfully. ${ }^{15}$ Severe preeclampsia, placental abruption and chorioamnionitis of the retained twin all have significant maternal and fetal risks. Additionally, caesarian section of the firstborn or other obstetrical indications for the delivery of all fetuses are also contraindications. However, selective hysterotomy of twin A with successful outcome in twin $B$ has been described. ${ }^{12,16}$ Oleyese et al. described that delayed delivery of twin B when the twin A is born at 24 weeks or later had no benefit to reducing mortality. The cohort study demonstrated that there was only a decrease in perinatal and infant mortality when twin A delivered at 22-23 weeks and when the delay interval was 3 weeks or less. Furthermore, delay intervals of 4 weeks or more were associated with a higher risk of small-for-gestational age birth in twin B, regardless of the gestational age of twin A. ${ }^{11}$

A large case series of 200 twin pregnancies in the United States from 1995-1998 described delayedinterval deliveries of the first twin born at 17-29 weeks with a mean delay of 6 days (range of 2-107 days). Delayed-interval delivery of twins compared to non-delayed twins at the same gestational age had a 1 -year survival rate of $56 \%$ compared to $24 \%$ for non-delayed twins. The case series concluded that delayed-interval deliveries could improve infant survival and reduce neonatal morbiditiy. ${ }^{17}$

Although slight intervals between the deliveries of siblings during critical gestational ages of less than 30 weeks can achieve clinically significant benefits and decrease both neonatal morbidity and mortality, the choice to do so should be up to the patient. Extending the gestation of the second sibling from previable to periviable age will reduce mortality, but it may also result in the risk of long-term morbidity due to prematurity. Therefore, the option to deliver both twins at the same age and time, with the risk of mortality, should be made available to the patient after significant discussion of risks and benefits. ${ }^{16}$ The critical period of perinatal morbidity and mortality is between weeks 23 and 28 of gestation. ${ }^{8}$

\section{Case}

A 37-year old G2P0 with dichorionic diamniotic twin pregnancy resulting from IVF presented at $23 \mathrm{w} 2 \mathrm{~d}$ to a high risk obstetrics centre after experiencing previous preterm premature rupture of membranes of twin A at 19 weeks gestation with persistent oligohydramnios. The ultrasound examination revealed cephalic presentation for twin A with transverse presentation for twin B. The patient had a remote history of anterior uterine fibroids for which she received a hysteroscopic myomectomy and was Group B Streptococcus negative.

The patient was treated with two doses of betamethasone at $23 \mathrm{w} 4 \mathrm{~d}$ and $23 \mathrm{w} 5 \mathrm{~d}$ and nifedipine at $25 \mathrm{w} 5 \mathrm{~d}$ for tocolysis due to extreme prematurity, during which she experienced a new onset of vaginal bleeding. At 25w6d she received $\mathrm{MgSO}_{4}$ for neuroprotection as she was felt to be in preterm labour. At 26w1d, twin A showed variable decelerations, and delayed interval delivery was presented as an option to the patient versus delivery of both fetuses at the same time. After extensive review of the literature and discussion of the maternal and neonatal risks and possible benefits, the decision to attempt the delayed interval delivery was agreed upon by the patient, her partner and the Maternal Fetal Medicine Department. The patient was afebrile, white blood count (WBC) was normal, and discharge was normal with no evidence of chorioamnionitis. Consequently, a spontaneous vertex delivery of a live male weighing $740 \mathrm{~g}$ with Apgar scores of 4, 5, 7 after 1, 5 and 10 minutes respectively, occurred in the operating room. The umbilical cord of twin A was clamped with two Endoloop PDS sutures and was cut close to the placenta. Nitroglycerine 200 $\mu \mathrm{g}$ IV was administered in addition to IV gentamicin and IV clindamycin as per recommendations in the literature search as nitroglycerine promptly initiates uterine quiescence. The lower uterine segment was then irrigated with 50000 units of bacitracin in $1 \mathrm{~L}$ of normal sterile saline, $700 \mathrm{cc}$ of which was flushed into the lower uterine segment through a 16 French Foley catheter. 
Following delivery of twin A, ultrasound examination of twin $B$ revealed a normal fetal heart rate (FHR) and cord presentation. The PDS suture was visible on ultrasound and twin $B$ was in transverse presentation. The patient was observed in the operating room with continuous electronic fetal monitoring for one hour. She was then started on indomethacin 100 mg PR for 48 hours and IV gentamicin and clindamycin was continued for 72 hours.

At $26 \mathrm{w} 2 \mathrm{~d}$, transvaginal ultrasound exam revealed a $3.2 \mathrm{~cm}$ long cervix and normal amniotic fluid volume around twin B (Figure 1). The interval delivery was delayed subsequently for 8 days. The delay period included daily $\mathrm{CBC}$ with C-reactive protein (CRP), fetal non-stress tests three times per day, broad-spectrum antibiotics for 7 days and tocolytics for 48 hours.

At 27w1d the patient was assessed for increased contractions every four to five minutes and a temperature of $37.7^{\circ} \mathrm{C}$. The patient had no loss of fluid or vaginal bleeding and FHR was normal. The pelvic exam demonstrated a closed cervix. One day later, blood and urine cultures were taken and IV gentamicin and clindamycin were restarted. The patient's temperature was $37.8{ }^{\circ} \mathrm{C}$ and heart rate was $120 \mathrm{bpm}$, while the FHR was $150-160 \mathrm{bpm}$ with moderate variability. With WBC $15.98 \times 10^{9} \mathrm{~L}^{-1}$ (bands 15\%), hemoglobin $10^{9} \mathrm{~g} \mathrm{~L}^{-1}$, platelets $280 \times 109 \mathrm{~L}^{-1}$, a diagnosis of chorioamnionitis was made. This resulted in delivery via low transverse caesarean section at $27 \mathrm{w} 2 \mathrm{~d}$ for breech presentation. A live male infant weighing $1100 \mathrm{~g}$ was delivered with Apgar scores of 6 and 8 at one and five minutes, respectively, with no signs of infection.

Pathology review revealed placenta A had focal acute funisitis and mild acute subchorionitis while placenta B had minimal focal acute phlebitis.

Post partum, the patient's WBC decreased to 10.91 $\mathrm{x} 10^{9} \mathrm{~L}-1$, hemoglobin $84 \mathrm{~g} / \mathrm{L}$ and both blood and urine

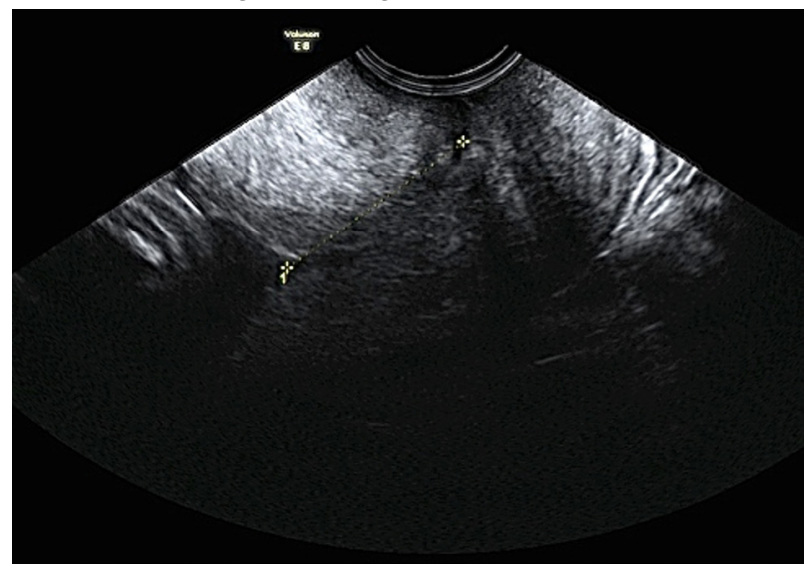

Figure 1. Transvaginal ultrasound at $26 \mathrm{w} 2 \mathrm{~d}$ demonstrating a clear internal os with a cervix measuring $3.2 \mathrm{~cm}$. There was no funneling or shortening with suprapubic or fundal pressure. cultures remained negative. The maternal post partum course was uneventful and she remained in hospital to care for her twins. Twin A initially required high frequency ventilation, then went onto conventional ventilation with low oxygen fraction for respiratory support. Twin B was initially intubated for surfactant administration, but quickly went onto continuous positive airway pressure. Both twins are currently in excellent condition and have returned home.

\section{Discussion}

While there are no guidelines regarding management following delivery of the first twin, high ligation of the umbilical cord with the placenta left in-situ is recommended. ${ }^{16}$ It is suggested that the umbilical cord be tied with absorptive suture close to the placenta. This allows the cord stump to be cut away from the placenta, which is thought to reduce the risk of chorioamnionitis. An Endoloop allows the suture to be placed higher in the birth canal, well above the internal os. ${ }^{18}$

Bacitracin $50000 \mathrm{U} / \mathrm{L}$ can then be applied to the lower uterine segment to reduce infection risk. It is important to monitor the presence of fever, leukocytosis and elevated CRP, as such markers may be indicative of chorioamnionitis. ${ }^{19}$ It is also recommended that ultrasonography is used to assess cervical dilation and length as digital pelvic examination can increase the risk of chorioamnionitis. ${ }^{20}$

Some study protocols suggest cervical cerclage after a seven day delay interval as silent cervical dilatation may be implicated in the preterm birth of twin A in the first place. Cerclage can minimize fetal membrane exposure to vaginal flora and low $\mathrm{pH} .{ }^{21}$ However, the placement of a cerclage prior to the decision to delay a delivery results in poorer outcomes, often due to intraamniotic infection, or rupture of membranes. Indeed, previous cerclage may even decrease the latency period, or period of delay between births, compared to a woman who has never had a cerclage. ${ }^{14}$ Cerclage may also be discouraged due to the possibility of increased risk of chorioamnionitis. ${ }^{22}$

The administration of tocolytics, in addition to broad-spectrum antibiotics, are also important interventions to consider when planning to delay the birth of a second fetus. Indeed, intra-amniotic infection is a common cause of failure to prolong pregnancy, as women who have intrauterine infection do not respond as well to tocolytic therapy. ${ }^{23}$

Many studies to date that report on outcomes of delayed-interval deliveries involved either case series or case reports. One review discussed the importance of delayed-interval deliveries that took the second sibling past the 24 th week, or the week of viability. ${ }^{8}$ Of such 
cases, 24/70 neonates survived and the range of latency was 3-143 days, with a mean latency of 44.8 days. Of the delayed deliveries that resulted in neonatal viability, the mean latency period was 57.5 days. However, delayed deliveries resulting in neonatal death corresponded to a mean latency of only 17.3 days. ${ }^{8}$

Maternal morbidity and mortality is also a primary concern with delayed-interval deliveries. A retrospective review of 5 sets of twins and 2 sets of triplets demonstrated that the duration of maternal hospital admission was directly correlated to length of delay between deliveries. This meant that the length of hospital admission was related to the prolonged antepartum course and not the post-partum stay due to possible maternal morbidity. Of the patients in the study, four experienced intra-amniotic infection and one had a case of maternal sepsis, which was treated with broad-spectrum antibiotics. There were no maternal deaths described. ${ }^{23}$ Another retrospective review referenced a patient who required a postpartum hysterectomy as a result of hemorrhage, uterine atony and myometrial micro-abscesses on pathology. The study also described a $31.6 \%$ incidence of serious maternal morbidity. ${ }^{12}$ Arabin and colleagues discuss the risks of chorioamnionitis (24\%), placental abruption (5\%), post partum hemorrhage (11\%) and manual removal of placental contents (11\%). They also describe a case of delayed interval delivery of triplets where the mother became septic from pyelonephritis. Furthermore, one patient went into transient atrial fibrillation without sequelae and another required an appendectomy three days post partum. ${ }^{22}$

\section{Conclusion}

Delayed-interval delivery provides an opportunity to increase the chance of survival of the second fetus in situations where the first is born too soon ( $<30$ weeks). Although there are no consensus guidelines on this approach, many case reports have suggested options that have provided positive outcomes that reduce neonatal morbidity and mortality.

It is paramount to ensure there are no signs of infection in the mother or fetuses before delay is considered and a thorough informed consent process must be undertaken as there is no robust research to support immediate delivery versus delayed interval delivery.

\section{Acknowledgements}

The patient whose case is presented has provided signed permission for its publication.

\section{References}

1. Martin JA, Hamilton BE, Sutton PD, Ventura SJ, Menacker F, Kirmeyer S, et al. Births: final data for 2006. Natl Vital Stat Rep 2009;57(7).

2. Lipitz S, Reichman B, Paret G, Modan M, Shalev J, Serr DM, et al. The improving outcome of triplet pregnancies. Am J Obstet Gynecol 1989;161(5):1279-84.

3. Ballard JL, Khoury JC, Wedig K, Wang L, Eilers-Walsman BL, Lipp R. New Ballard Score, expanded to include extremely premature infants. J Pediatr 1991;119(3):417-23.

4. American College of Obstetricians and Gynecologists Committee on Practice Bulletins-Obstetrics, Society for Maternal-Fetal Medicine, ACOG Joint Editorial Committee. ACOG Practice Bulletin \#56: Multiple gestation: complicated twin, triplet, and high-order multifetal pregnancy. Obstet Gynecol 2004;104(4):869-83

5. "Multiple Birth". SOGC website <http://sogc.org/publications/multiplebirth/> (accessed January 5, 2014).

6. Russell RB, Petrini JR, Damus K, Mattison DR, Schwarz RH. The changing epidemiology of multiple births in the United States. Obstet Gynecol 2003;101(1):129-35.

7. Lambalk CB, De Koning CH, Braat DD. The endocrinology of dizygotic twinning in the human. Mol Cell Endocrinol 1998;145(1-2):97-102.

8. Platt JS, Rosa C. Delayed interval delivery in multiple gestations. Obstet Gynecol Surv 1999;54(5):343-8.

9. Hamersley SL, Coleman SK, Bergauer NK, Bartholomew LM, Pinckert TL. Delayed-interval delivery in twin pregnancies. J Reprod Med 2002;47(2):125-30.

10. Fanaroff AA, Wright LL, Stevenson DK, Shankaran S, Donovan EF, Ehrenkranz RA, et al. Very-low-birth-weight outcomes of the National Institute of Child Health and Human Development Neonatal Research Network, May 1991 through December 1992. Am J Obstet Gynecol 1995;173(5):1423-31.

11. Oleyese Y, Ananth CV, Smulian JC, Vintzileos AM. Delayed interval delivery in twin pregnancies in the United States: impact on perinatal mortality and morbidity. Am J Obstet Gynecol 2005;192(2):439-44.

12. Roman AS, Fishman S, Fox N, Klauser C, Saltzman D, Rebarber A. Maternal and neonatal outcomes after delayed-interval delivery of multifetal pregnancies. Am J Perinatol 2011;28(2):91-6.

13. Synnes AR, Buchanan L, Ruth C, Albersheim S. Management of the newborn delivered at the threshold of viability. BCMJ 2008;50(9):498-508.

14. Farkouh LJ, Sabin ED, Heyborne KD, Lindsay LG, Porecco RP. Delayedinterval delivery: extended series from a single maternal-fetal medicine practice. Am J Obstet Gynecol 2000;183(6): 1499-503.

15. Beinder E, Lang N. Delayed interval delivery in a twin pregnancy with monochorionic placenta. Am J Obstet Gynecol 1997;176(1 Pt 1):254.

16. Porreco RP, Sabin ED, Heyborne KD, Lindsay LG. Delayed-interval delivery in multifetal pregnancy. Am J Obstet Gynecol 1998;178(1 Pt 1):20-3.

17. Zhang J, Hamilton B, Martin J, Trumble A. Delayed interval delivery and infant survival: a population-based study. Am J Obstet Gynecol 2004;191(2):470-6.

18. Surico D, Amadori R, Ferrero F, Vigone A, Leo L, Surico N. Dichorionic pregnancy: delayed interval delivery with endoloop ligation. Twin Res Hum Genet 2012;15(4):537-40.

19. Van Doorn HC, van Wezel-Meijler G, van Geijn HP, Dekker GA. Delayed interval delivery in multiple pregnancies; is optimism justified? Acta Obstet Gynecol Scand 1999;78(8):710-5.

20. Wittmann BK, Farquharson D, Wong GP, Baldwin V, Wadsworth LD, Elit L. Delayed delivery of second twin: report of four cases and review of the literature. Obstet Gynecol 1992;79(2):260-3.

21. Zhang J, Johnson CD, Hoffman M. Cervical cerclage in delayed interval delivery in a multifetal pregnancy: a review of seven case series. Eur J Obstet Gynecol Reprod Biol 2003;108(2):126-30.

22. Arabin B, van Eyck J. Delayed-interval delivery in twin and triplet pregnancies: 17 years of experience in 1 perinatal center. Am J Obstet Gynecol 2009;200(2):154.e1-8.

23. Kalchbrenner MA, Weisenborn EJ, Chyu JK, Kaufman HK, Losure TA. Delayed delivery of multiple gestations: maternal and neonatal outcomes. Am J Obstet Gynecol 1998;179(5):1145-9. 